cost-effectiveness of preoperative autologous blood donations. N Engl J Med 1995;332(11):719-722.

\section{Pertussis Found to Be a Common Cause of Persistent Cough in Adults}

There still is a common perception that pertussis is a disease primarily of children. Increasing evidence indicates that adults play a predominant role in the transmission of pertussis and may be the main reservoir of the disease. Dr. Seth Wright and colleagues from Vanderbilt University Medical Center in Nashville, Tennessee, recently studied the prevalence of pertussis in 75 adult patients presenting to the hospital emergency department with a chief complaint of cough of at least 14 days' duration. The mean age of the patients was 32 years, and the average duration of cough was 26 days (range, 14 to 90 days). All but one had been vaccinated against pertussis as a child. One had childhood pertussis, and none were known to have had pertussis as an adult.

Acute and convalescent antibody levels against pertussis toxin and filamentous hemagglutinin were determined by ELISA. Sixteen of the 75 patients had serologic evidence of pertussis, including 13 who had elevated levels on presentation. Cultures were negative in all 75 patients.

Clinical symptoms (paroxysmal cough, posttussive emesis, and fever) and lymphocyte count did not differentiate patients with pertussis from those without the disease.

The results of this study confirm that pertussis infection may be common among adults with persistent cough and that clinical signs and symptoms, pertussis culture, DFA testing, and lymphocytosis are of limited value in making the diagnosis. The authors suggest that vaccination of adults with the newly developed and less reactive acellular vaccines may be indicated if other studies confirm the high incidence of pertussis in adults.

FROM: Wright SW, Edwards KM, Decker MD, Zeldin MH. Pertussis infection in adults with persistent cough. JAMA 1995;273:1044-1046.

\section{Zaire's Ebola Outbreak Coming Under Control}

With the slowing of new cases of Ebola viral hemorrhagic fever (VHF), health officials from the World Health Organization recently announced that the epidemic was coming under control. More than 160 suspected or confirmed cases have been identified, of which 121 have been fatal. Cases have included hospital workers that cared for infected patients. The majority of cases were from Kikwit, Zaire, a city of approximately 600,000 , located 240 miles east of Kinshasa, the capital of Zaire. Thus far, no cases have been reported from Kinshasa. It is believed that the index case for this outbreak was a forest worker infected in December 1994. Members of the international team have expressed hope that clues from the index case may reveal the currently unknown reservoir for this virus.
Transmission of the virus to secondary cases occurs through close personal contact with infectious blood or other body fluids or tissue. In previous outbreaks, secondary cases occurred among persons who provided medical care for patients and among patients exposed to reused needles. Although aerosol spread has not been documented among humans, this mode of transmission has been demonstrated among nonhuman primates. Because of the high fatality rate and lack of specific treatment or a vaccine, work with this virus in the laboratory setting requires biosafety level 4 containment.

The incubation period is from 2 to 21 days. Initial clinical manifestations of Ebola hemorrhagic fever include fever, headache, chills, and myalgia; subsequent manifestations include severe abdominal pain, vomiting, and diarrhea. Hemorrhagic manifestations with presumptive disseminated intravascular coagulation usually occur in fatal cases. In reported outbreaks, $50 \%$ to $90 \%$ of the cases have been fatal.

The Ebola virus takes its name from the Ebola River area in northern Zaire, where the first documented outbreak of this disease occurred in 1976. Ebola virus and Marburg virus are the two known members of the filovirus family. Ebola viruses first were isolated from humans during concurrent outbreaks of VHF in northern Zaire and southern Sudan in 1976. An earlier outbreak of VHF caused by Marburg virus occurred in Marburg, Germany, in 1967, when laboratory workers were exposed to infected tissue from monkeys imported from Uganda. Two subtypes of Ebola virus, Ebola Sudan and Ebola-Zaire, previously have been associated with disease in humans. In 1989, an outbreak among monkeys imported into the United States from the Philippines was caused by another Ebola virus, which infected humans but was not associated with clinical disease. This 1989 US outbreak is the subject of a popular new book, The Hot Zone, by Richard Preston (Random House).

CDC has established a hotline for public inquiries about Ebola virus infection and prevention, (800) 900-0681. CDC and the state department have issued travel advisories for persons considering travel to Zaire. Information about travel advisories to Zaire and for air passengers returning from Zaire may be obtained from the CDC International Travelers' Hotline, (404) 332-4559.

FROM: CDC. Outbreak of Ebola viral hemorrhagic fever-Zaire, 1995. MMWR 1995;44(19):381-382.

Altman L. UN agency says worst may be over in Zaire's Ebola epidemic. N ew York Times May 27, 1995, p A6.

Additional news items in this issue: Hospital Epidemiology Training Course, page 380; Blood Culture Results Found to Have Limited Effect on Antibiotic Choice by House Staff, page 381; OSHA's TB Standard to Be Peer Reviewed, page 384; FIASH! Late-Breaking News, page 384; OSHA Updates Guidelines on Hazardous Drug Exposures, page 390; Risk of HIV Transmission from Healthcare Worker to Patient Is Very Small page, 404; CDC Reports a Decline in TB Cases for Second Year in a Row, page 411; CDC Offers 24-Hour Fax-Back of HIV Documents, page 4 16; Nursing Home Fined $\$ 75,000$ for Isolating Patient for 9 Months, page 418. 